\title{
The optimization of curriculum design of electronic technology (CDET) at Chinese engineering colleges
}

\author{
SU Guo-jun ${ }^{1, a}$, ZHENG Tong ${ }^{2, b}$, ZHANG Cui-min ${ }^{3, c}$, WANG Jin ${ }^{4, d}$ \\ ${ }^{1,2,3}$ TianJin Keylaboratory of Information Sensing\&Intelligent Control, Tianjin University of technology \\ and education, China \\ ${ }^{4}$ School of Information Technology Engineering, Tianjin University of technology and education, \\ China \\ aguojunsu@163.com, bzheng_tong@126.com, czhangcuimin@126.com, dwangjin800505@163.com
}

Keywords: curriculum design of electronic technology (CDET); engineering colleges; topic selection; teaching method; simulation; design report

\begin{abstract}
The curriculum design of electronic technology (CDET) is one of the most important practice links at Chinese engineering colleges. It is critical to deepen understanding of the application of the premise courses (such as Analog Electronic Technology and Digital Electronic Technology), and at the same time, it started laying the groundwork for the follow-up related courses, graduation design and even graduation work. In order to improve the quality of the CDET, the main links of it such as topic selection, the teaching method, simulation and debugging, and curriculum design report, are optimized and explored.
\end{abstract}

\section{Introduction}

"The course design of electronic technology (CDET)" is a compulsory course for electrical and control engineering majors, and CDET is also an important link of practice teaching for related professional students. Basic electronic product design knowledge and skills can be grasped through the CDET, at the same time, it can train students' professional learning enthusiasm and cultivate good professional quality, which lays a solid foundation for subsequent courses and social work after graduation .

The course has two basic tasks: one is to deepen the course knowledge of "Analog Electronic Technology" , "Digital Electronic Technology", and the experimental skills of electronic technology; and the other is to improve the practical skills in electronic technology and the ability of scientific research, which also cultivates students' ability of innovation and team cooperation. Through the systematically CDET training, the procedures of similar electronic engineering design and the other same scientific practices will be well done in the future .

To improve the quality of teaching and learning, the research group always optimize the process of the CDET to highlight the dominant position of students in the process of curriculum design and cultivate students' practical ability and innovation ability[1-3].

\section{Material and Method}

Optimize Design Topic. The appropriate topic of design can stimulate students' interest and improve students' enthusiasm , the above inner learning factors can not only improve the teaching effect, but also can make teaching and learning in joy. The principles of topic selection are rich content, practical, advanced, innovation and interest. The difficulty of the design topic should be moderate, but which should can reflect the comprehensive application of the basic theory, basic concepts and basic skills (known collectively as 3B). Therefore, the design topic should be a complete system of comprehensive application of Analog Electronic Technology and Digital Electronic Technology. At the same time, the class hours of design should be arranged reasonably, and the design should include compulsory topic and optional topic, the compulsory topic is mainly to review and enhance students' understanding of basic knowledge of electronics, at the same time, teachers and students can make a horizontal comparison to improve the overall design ability of 
electronic technology, the optional topic is mainly to improve learning enthusiasm and innovation ability of students, through the combination of compulsory topic and optional topic as the design topic, the CDET can consolidate learned electronic technology, and cultivate students' learning enthusiasm, both of which lay a solid foundation for the subsequent courses.

The Project-based and Modules Design Strategy. About the traditional teaching mode, the CDET is elaborated in every detail including design steps, and the students in the whole design process rarely think about the CDET independently, let alone question and exploration. Different from above traditional teaching mode, the project-based learning[4-5] method is used in the whole design process, the CDET is only guided by teachers, and the students complete the specific project cases by themselves. According to project-based learning method, the students' exploration spirit and practice ability are improved obviously. The CDET development process simulates the above actual design process including the project task, program settings, software simulation and hardware debugging etc.. The CDET should include following links : select design title ,make task book, determine the design scheme, circuit simulation, electronic components of the electronic system design, installation and debugging , and write the CDET report .

In order to improve students' innovation ability, emphasize students' independent design and pay more attention to the heuristic teaching, the CDET teachers guide only in the general idea, and do not intervene too much on the concrete scheme and design details, sometime except the organization, guidance, inspection, check and solve some difficult problems. Give full play to the initiative and creativity of students. At the same time, in order to coordinate with the proposed CDET method, a complete and rich variety of components are provided for the students to choose.

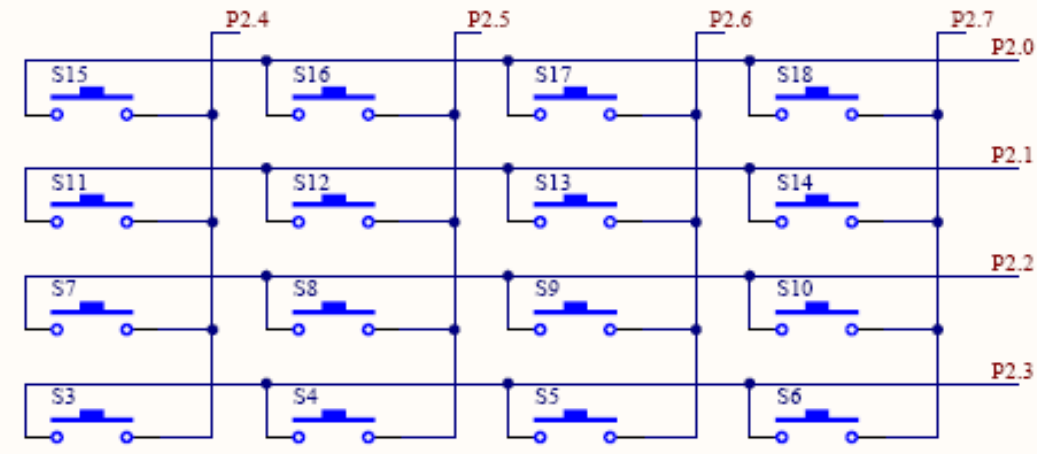

Fig.1. 4*4 Keyboard Module

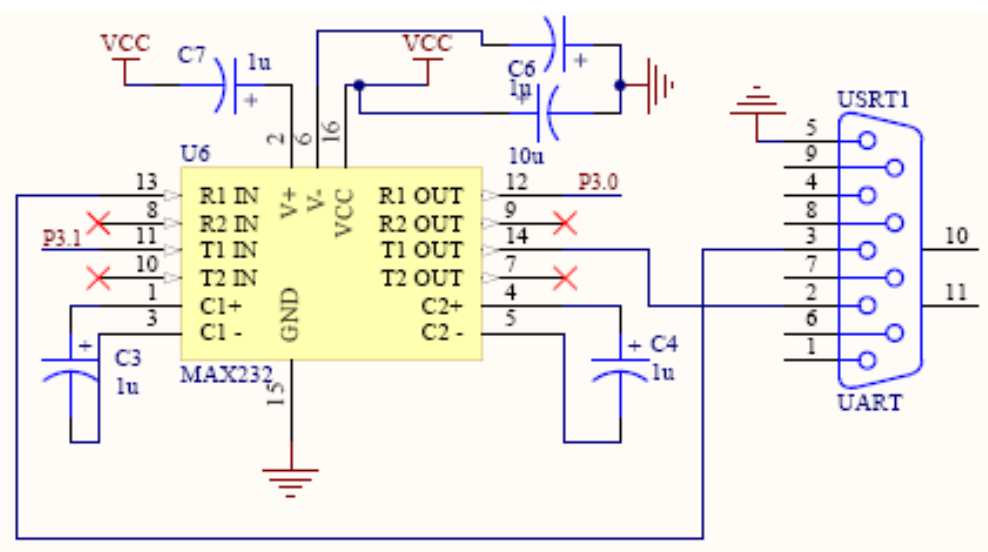

Fig.2. Level Conversion Module

With the development of electronic industry, the design of electronic products in all aspects is more and more complex. In order to adapt to the development trend, the students should be suggested that the whole CDET should break up into several modules to reduce the difficulty, such as frequently-used $4 * 4$ keyboard module (Fig.1), level conversion module (Fig.2) and so on. First, single module is designed and debugged independently, then the combination of several 
modules is debugged, and ultimately the whole CDET system is debugged. The above method provides the effective design strategy for the more complex electronic systems[6-7, 9].

The EDA for Design and Simulation. With the rapid development of computer technology, circuit design and simulation using EDA software are a trend. EDA has been introduced to practical teaching reform in many colleges and universities, and EDA laboratories are established accordingly.The laboratories are equipped with some powerful tools, such as EWB, Pspice, Multisim, Protel, MaxPlusII, SystemView and other related software and hardware. Multisim is the main simulator of some separation devices and their combination; circuit simulation can be performed by Protel, but its function is not very strong, and which is mainly used to design the circuit board; Matlab is powerful for the analysis of a large comprehensive mathematical computing platform. At present, Proteus has been widely used in the world, thousands of colleges including Stanford and Cambridge take Proteus as teaching and experiment platform for concerned electronic courses.

Proteus developed by Labcenter Electronics is a famous EDA tool, which not only has the function of the simulation software like other EDA tools, but also can simulate the electronic system with MCU and peripheral devices, such as Fig.3.

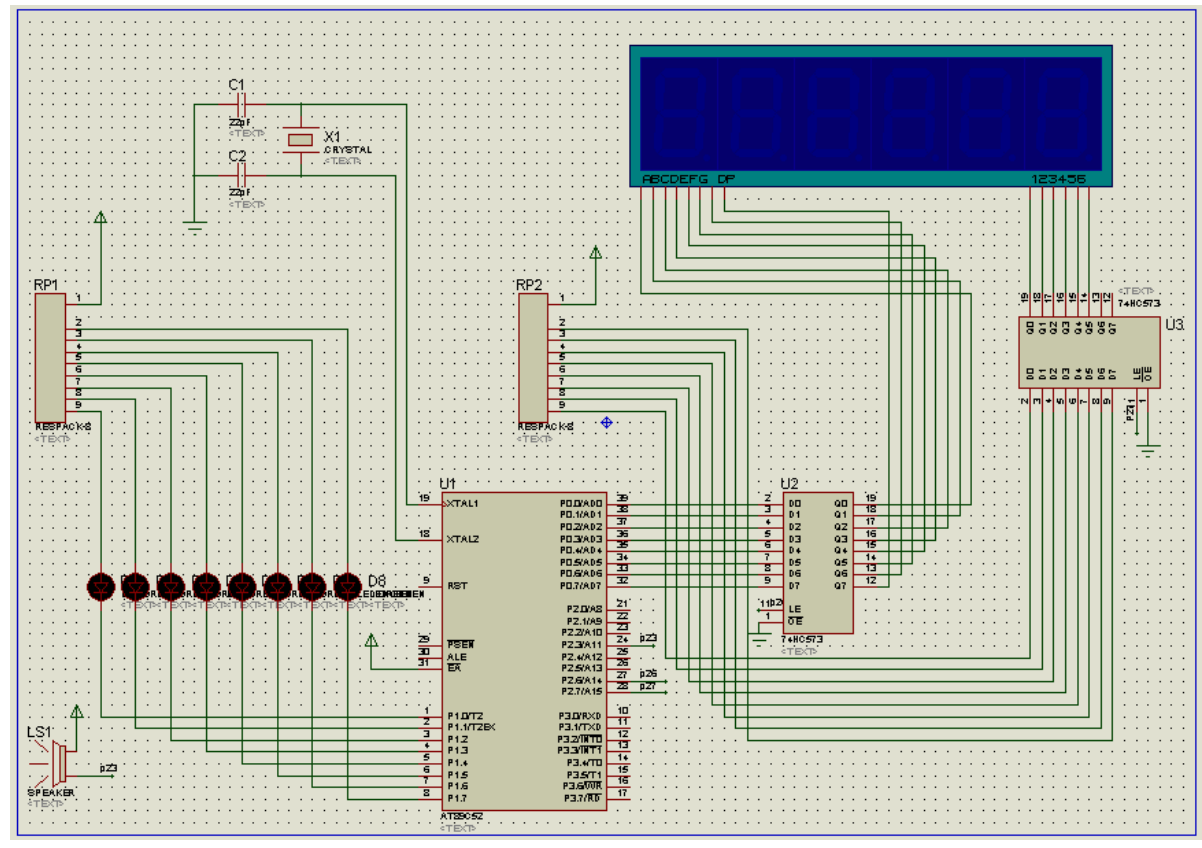

Fig.3. Example of Proteus Simulation

Proteus realizes the complete design from concept to product, including the functions such as the schematic layout, code debugging, co-simulation of the micro-controller and peripheral circuit , and a key switch to PCB design. Proteus is currently the world's only united design platform of circuit simulation software, PCB design software and virtual simulation software. The processor model of Proteus supports the 8051, PIC, AVR, ARM, 8086 ,MSP430, Cortex, DSP series processors and etc. About the compiler, Proteus supports IAR, Keil, MPLAB and other compilers.

The Writing of CDET Report. Writing CDET report is a very important link , the report should be in accordance with the requirements of scientific paper. To write the CDET report carefully can not only cultivate the students' rigorous and realistic style of scientific research, but also can lay a foundation for the future writing of graduation design report, scientific papers, and so on.

The CDET report is composed of the following parts:

(1) The CDET report title and requirements;

(2) The CDET program demonstration and determination;

(3) Design, parameter calculation and component selection of the CDET system ;

(4) Module combination and system debugging;

(5) The rest of the content, such as components list, reference, etc. 
The students maybe have few opportunities to write such a report, so teachers should guide students to write the general outlines of their reports, then let the students filling content according to general outlines. In particular, the CDET report is a whole process, there should be more detailed analysis of the CDET.

Establishing a Scientific CDET Evaluation System. Establishing a scientific evaluation system and reasonable evaluation of students' CDET achievement are important factors to ensure the quality of CDET, at the same time, which can fully mobilize the enthusiasm of the students. It is necessary for CDET teachers to consider the quality of process and the actual situations of the theory and practical problems, students with the creative thinking should be encouraged in the evaluation of students' CDET achievement.

The curriculum design is different from theoretical courses which is easy to evaluate the students' academic achievement, such as test paper. The CDET achievement is a fuzzy evaluation, including all stages of design. To make a reasonable evaluation standard, teachers should decide the weight and criteria at various stages of CDET through consultation. At the same time, the teachers should encourage students to innovate and seriously study the issue through the formulated evaluation standard, as long as the students have their own research content, characteristics and innovation, even if some ideas failed to achieve, or there are some problems in the implementation, the teachers should give good evaluation to encourage the spirit of exploration. Only by this way, students' enthusiasm and good learning atmosphere can be established continuously[8-9].

\section{Summary}

Through the survey of discussion and questionnaire about CDET, results showed that the students' understanding of the related courses is more profound through the CDET, and the students are more interesting and confident of other curriculum designs and their coming graduate designs. Chinese engineering colleges should make more research and exploration on the basis of the concerned experience, and improve constantly the CDET content and method, only by this way, the students' practical ability, comprehensive ability and innovation ability can be improved.

\section{Acknowledgment}

This work was financially supported by the Scientific Research Foundation of Tianjin University of Technology and Education (KYQD12014).

\section{References}

[1] Z.Z. Jiang, The Combination of Innovation Education and Electronic Technology Teaching: Journal of Chongqing Polytechnic College,6 (2004)

[2] Y. Wu, Discussion on practice course construction reform of "Electronic Technology": Journal of Changsha Telecommunications and Technology Vocational College, 1(2011)

[3] X.B.Hu,J.F.Li The Application of CAI in the Teaching Modernization of Electronic Technology Class: Computer Knowledge and Technology, 21(2008)

[4] J.M. Guarasa, J.M.Montero.etc. A Project-Based Learning Approach to Design Electronic Systems Curricula: IEEE Trans. Educ., 3(2006)

[5] D.S. Martin, J.A.Martinez.etc. Problem-Based Learning in Wind Energy Using Virtual and Real Setups: IEEE Trans. Educ., 1(2012)

[6] J.J.Yu,J.F.Qiao Teaching Practice of Course Design for Automatic Control System Based on Task Driving: Research and Exploration in Laboratory, 11(2013) 
[7] S.T.Li,J.Zhang.etc. The Reform and Innovation of Research-Oriented Teaching Mode of Electronics and Information Courses: Journal of Higher Education Research, 4(2011)

[8] H.L.He. Study on the integration of electronic application courses design: Education Forum, $1(2014)$

[9] X.J.Zhang. The development of theme oriented electronic technology course teaching: Vocational and Technical Education, 5(2014) 